Marquette University

e-Publications@Marquette

Clinical Lab Sciences Faculty Research and

Publications

Clinical Lab Sciences, Department of

2018

\title{
A Preliminary Investigation of Acculturative Stress and Diurnal Cortisol Among Latina Women
}

Lucas Torres

Felicia Mata-Greve

April L. Harkins

Follow this and additional works at: https://epublications.marquette.edu/clinical_lab_fac

Part of the Laboratory and Basic Science Research Commons 
Marquette University

e-Publications@Marquette

\section{Clinical Laboratory Science Faculty Research and Publications/College of Health Sciences}

This paper is NOT THE PUBLISHED VERSION; but the author's final, peer-reviewed manuscript. The published version may be accessed by following the link in th citation below.

Journal of Latina/o Psychology, Vol. 6, No. 2 (2018): 149-158. DOI. This article is @ American Psychological Association and permission has been granted for this version to appear in $\underline{\mathrm{e}}-$ Publications@Marquette. American Psychological Association does not grant permission for this article to be further copied/distributed or hosted elsewhere without the express permission from American Psychological Association.

\section{A Preliminary Investigation of Acculturative Stress and Diurnal Cortisol Among Latina Women}

\section{Lucas Torres}

Department of Psychology, Marquette University;

Felicia Mata-Greve

Department of Psychology, Marquette University

April Harkins

Clinical Laboratory Science Department, Marquette University

\footnotetext{
Abstract

The interplay between biological and cultural factors has remained understudied among ethnic minority groups, including Latinos. To the best of our knowledge, minimal to no research has examined the relationship between acculturative stressors and diurnal cortisol among Latina women. This initial exploratory study sought to examine the relationship between cortisol and acculturative stress. Among a small sample of adult Latina women, salivary cortisol was collected at 3 time points (waking, 30 min postwaking, and bedtime) to assess the
} 
cortisol awakening response (CAR) and diurnal cortisol response. Information regarding acculturative stress and acculturation was also collected. The major results showed that high levels of acculturative stress were associated with a blunted CAR and a flatter diurnal cortisol response when compared to low levels of acculturative stress. These preliminary findings highlight the potential role of cultural stressors in contributing to the biological stress response.

\section{Keywords:}

Latino ental health, acculturative stress, diurnal cortisol, cortisol awakening response

Stressful life events have often been linked to poor mental health through a dysregulation of physiological systems such as the hypothalamic-pituitary-adrenal (HPA) axis and the main stress hormone cortisol (Juster, McEwen, \& Lupien, 2010). Unfortunately, marginalized groups have benefited less than other groups from scientific advancements in the knowledge of biological pathways and physiological responses to stress (Clingerman \& Brown, 2012). Some work has sought to examine the biological factors associated with cultural stressors-primarily ethnic discrimination and mental health (Myers, 2009; Pascoe \& Smart Richman, 2009). Generally speaking, these models posit that exposure to cultural stressors, particularly when chronic or long lasting, results in physiological changes that in turn contribute to psychological and physical problems. However, minimal to no research has investigated the relationship between cortisol and acculturative stress within Latino groups.

As one of the fastest growing ethnic groups in the United States, Latinos constitute approximately $17 \%$ of the population, and projections estimate that the number will rise to $30 \%$ of the population by 2050 (U.S. Census Bureau, 2013). Regardless of nativity status or time spent living in the United States, Latinos are likely to experience acculturative stress. A recent survey revealed that women across ethnicities reported higher average stress levels when compared to men and indicated that their stress levels had increased in the previous year (American Psychological Association, 2016). Perhaps similar to other ethnic groups, women of Latino descent are likely to experience additional stressors related to family and cultural expectations (Galanti, 2003). As such, investigating the experiences of Latina women will provide important insight into the health of this underrepresented group. This study sought to provide an initial exploratory examination of the relationship between cortisol and acculturative stress among Latina women.

\section{Cortisol and Stress}

The HPA axis is one of the body's main stress response systems. During instances of acute stress, the HPA axis releases corticotropin-releasing factor (CRF) in the hypothalamus, which in turn activates the pituitary gland to release adrenocorticotropic hormone (ACTH). ACTH then stimulates the adrenal cortex to release cortisol, a main stress hormone (Jackson, Knight, \& Rafferty, 2010; McEwen, 1998). Whereas acute responses to stress are considered adaptive, chronic activation of the HPA axis from cumulative stress can lead to dysregulation of the system (Dowd, Simanek, \& Aiello, 2009). The role of the HPA axis in response to stress is complex and dynamic in nature. Still, salivary cortisol is commonly used as an indicator of HPA functioning (Kudielka \& Kirschbaum, 2005). Cortisol is also thought to be a critical biological intermediary linking chronic stressors to physical and mental health problems (Miller, Chen, \& Zhou, 2007). The natural diurnal rhythm is such that cortisol levels show a 50 to $75 \%$ rise within the first 30 to 45 min after waking. Following this morning peak, cortisol levels drop sharply and then decrease more gradually throughout the day, reaching their lowest point in the evening around bedtime (Pruessner et al., 1997). 
The cortisol awakening response (CAR) refers to the changes in cortisol levels experienced between waking and the morning peak (30 to 45 min after waking). Although CAR dysregulation has been used in the past as an indicator of stress-induced injury to the HPA axis (Pruessner et al., 1997), more recent research has postulated that the CAR is a distinct and independent process from diurnal patterns in HPA activity (Clow, Hucklebridge, Stalder, Evans, \& Thorn, 2010). The exact function of the CAR remains unclear but has been linked to the anticipation of demands and perceived burden (Fries, Dettenborn, \& Kirschbaum, 2009). Among Mexican American adults, an attenuation of the CAR-or smaller differences between waking and postwaking cortisol levels - has been associated with subclinical symptoms of depression (Mangold, Marino, \& Javors, 2011).

Generally speaking, diurnal rhythms characterized by decreased morning levels and increased afternoon or evening cortisol, resulting in flatter slopes, are indicative of greater HPA dysregulation and mental health symptoms (Skinner, Shirtcliff, Haggerty, Coe, \& Catalano, 2011). Past research has reported flatter diurnal cortisol rhythms among ethnic minorities, primarily African Americans, when compared to non-Hispanic Whites (Adam et al., 2015; Skinner et al., 2011). A similar pattern has emerged for Latina women who showed a flatter cortisol response pattern than non-Hispanic women (Gallagher-Thompson et al., 2006).

\section{Acculturative Stress}

The process of adapting to two or more cultures, referred to as acculturation, has been described as inherently stressful. As such, acculturative stress refers to the demands that stem from intercultural contact or the process of cultural adaptation (Berry, 2006). Individuals who interact with a new culture have to negotiate varying, and at times opposing, sets of values and expectations (Gonzales, German, \& Fabrett, 2012). Acculturative stressors can include pressures from both the mainstream U.S. culture and the traditional culture (Rodriguez, Myers, Mira, Flores, \& Garcia-Hernandez, 2002). Some examples of acculturative stress can include learning a new language, adhering to different cultural norms, retaining the native language and customs, and brokering between American and Latino ways of living (Torres, Driscoll, \& Voell, 2012). Acculturative stress has been linked to poor Latino mental health outcomes in numerous studies (

As mentioned previously, research examining cortisol and acculturative stress is scarce. Some work has investigated the relationship between cortisol and acculturation among Latino samples. For example, research with a sample of pregnant Latina women reported that high levels of acculturation predicted increased total cortisol levels (Ruiz, Pickler, Marti, \& Jallo, 2013), whereas reduced HPA activity was noted among later generation participants (Ruiz, Stowe, Brown, \& Wommack, 2012). In contrast, another study indicated that increased time in the United States was associated with blunted total cortisol output among Latino men ( $\underline{\text { Squires }}$ et al., 2012). Among Mexican American men, high levels of adherence to the Anglo or mainstream culture were associated with a decrease in the CAR (Mangold, Mintz, Javors, \& Marino, 2012; Mangold, Wand, Javors, \& Mintz, 2010). The authors of these studies conclude that acculturative stress is a significant factor in CAR dysregulation.

Research examining diurnal cortisol patterns with Latinos reported that among pregnant women of Mexican descent, greater acculturation was associated with a flatter slope (D’Anna-Hernandez et al., 2012). Among Latino day laborers, high allostatic load-an indicator of physiological changes due to chronic stress-was associated with greater everyday discrimination, being questioned about legal status, and fear of being deported (de Castro, Voss, Ruppin, Dominguez, \& Seixas, 2010). In contrast, a study with pregnant women of Hispanic origin found no relation between cortisol levels and cumulative stress, as measured by interpersonal violence, community violence, and ethnic discrimination (Suglia et al., 2010). This particular study did not assess acculturative stress. Overall, inconsistencies exist in the empirical literature regarding the nature of cortisol dysregulation in relation to cultural variables among Latinos living in the United States. Some of these discrepancies can be attributed to the methodological differences by which biological stress and cortisol are 
measured (i.e., total cortisol output vs. diurnal response), along with the minimal studies that have accounted for cultural variables in a systematic manner.

This study sought to address the gaps in the empirical literature examining biological factors and cultural variables-namely, acculturative stress-within Latino groups. As such, the purpose was to investigate the relationship between cortisol and acculturative stress in a sample of Latina women. Hypothesis 1 indicated that high levels of acculturative stress would be associated with a diminished CAR when compared to low acculturative stress. Hypothesis 2 stated that while controlling for acculturation and age, significant differences would emerge in the patterns of diurnal cortisol between low and high levels of acculturative stress. It was expected that high levels of acculturative stress would be associated with markers of dysregulation-namely, a flatter diurnal slope.

\section{Method}

\section{Participants and Procedures}

This study included 18 adult women who self-identified as Latina or Hispanic. The average age of the sample was approximately thirty-six years $(S D=10.59$, range of 24-54). In terms of nativity status, 12 individuals reported having been born outside of the United States. The majority of participants reported having obtained a bachelor's degree or higher $(n=13)$, with the remaining having some college and/or a high school degree or equivalency. Participants were asked to choose from categories of annual household incomes (i.e., less than $\$ 35,000, \$ 35,000$ to $\$ 50,000$, and greater than $\$ 50,000$ ) as an indication of socioeconomic background. Five participants reported an annual income of less than $\$ 35,000$, eight indicated having an annual income of between $\$ 35,000$ and $\$ 50,000$, and six reported earning more than $\$ 50,000$ annually. In the region where participants were recruited, the median annual household income for Latinos was $\$ 36,121$ (Center for Economic Development, 2016).

All study procedures were approved by the host university's institutional review board. Participants were recruited at a local community center with the assistance of key personnel within the community center who served as liaisons between the researchers and the participants. Trained bilingual (English-Spanish) research assistants obtained informed consent and explained the study procedures. Once individuals had provided consent to participate in the study, they were asked to complete a packet of questionnaires (see the Measures section) in their preferred language. The majority of respondents filled out the surveys in English ( $n=14 ; 78 \%$ ). Upon completion of the questionnaires, the research assistants distributed cortisol kits and described their contents and the collection protocol. Participants were instructed to begin the protocol of collecting salivary cortisol the next day or the following Monday to ensure that sampling took place on a weekday. The participants then arranged to deliver the salivary cortisol samples to the research assistants at the community center. Participants were given $\$ 10$ gift cards for completion of the questionnaires and $\$ 30$ gift cards for the salivary cortisol samples.

\section{Measures}

\section{Acculturative stress}

The Multidimensional Acculturative Stress Inventory (MASI; Rodriguez et al., 2002) is a 36-item instrument that assesses an individual's level of acculturative stress related to competency of English and Spanish language use, pressures to acculturate to the mainstream culture, and pressures against acculturation to the mainstream culture. Respondents rate items according to the previous 3 months on a 6-point scale ranging from 0 (does not apply) to 5 (extremely stressful). Items are averaged to obtain an overall score that ranges from 0 to 5 , where higher MASI scores correspond to greater acculturative stress. As reported previously (Rodriguez et al., 2002), 
the MASI is a valid and reliable measure, with a Cronbach's alpha of .91 in the current sample (U.S. born: .92, foreign born: .92).

\section{Acculturation}

The Brief Acculturation Rating Scale for Mexican Americans-II (BARSMA-II; Bauman, 2005) is a 12-item questionnaire available in English and Spanish adapted from the original 30-item Acculturation Rating Scale for Mexican Americans (ARSMA; Cuéllar, Arnold, \& Maldonado, 1995). The measure includes an Anglo Orientation subscale (AOS) and a Mexican Orientation subscale (MOS). Participants indicate how often they endorse the statement on a 5-point Likert scale that ranges from 1 (not at all) to 5 (extremely often or almost always). Responses are averaged and can range from 1 to 5; higher scores indicate stronger self-reported adherence to the respective culture. Cronbach's alphas for this study were 0.90 for the AOS and 0.81 for the MOS.

\section{Salivary cortisol}

Free cortisol levels were measured from salivary samples that were gathered at three specific time points (upon waking, 30 min postwaking, and before going to bed) as suggested previously (Adam \& Kumari, 2009). Free cortisol is recommended for the assessment of dynamic HPA axis activity (Gozansky, Lynn, Laudenslager, \& Kohrt, 2005; Kudielka, Hellhammer, \& Wüst, 2009). Participants were asked to avoid drinking alcohol (12 hr prior), eating (60 min prior), smoking, and brushing teeth before the collection of samples. Participants were instructed to wash or rinse their mouths for 2 to 3 min prior to sample collection. Participants were then to "chew" on a cotton swab for 30 to $40 \mathrm{~s}$ and place the swab in a plastic tube (Salimetrics LLC, State College, PA), which they were then to label with the time and date. Participants were strongly encouraged to report accurate times of sampling even if the protocol was not followed. They were instructed to store the samples in a freezer as soon as possible until they could be delivered to the research assistants. Once retrieved by the research assistants, the salivary samples were immediately transported and stored in an environment of $-20^{\circ} \mathrm{C}$ for later analysis. Overall, samples were exposed to room temperatures for a minimal amount of time during transport from participants' homes to the community center to the university laboratory for storage (typically 20-60 min).

Although menstrual cycle phase and oral contraceptives have been found to influence HPA activity (Kudielka \& Kirschbaum, 2005), a community liaison to this study suggested that gathering this information could be perceived as inappropriate within the community. Thus, these data were not gathered to respect the cultural norms of this group. The viability of the specimens was examined prior to analysis, along with the times the samples were taken. Postwaking cortisol samples were collected within $45 \mathrm{~min}$ of the waking samples (median of $30 \mathrm{~min}$ ). One participant was not able to follow this protocol (postwaking sample was 90 min after waking) and was excluded from the main analyses. Salivary cortisol levels were quantified using an enzymatic immunoassay (Salimetrics LLC, State College, PA). Previous studies have established the reliability of this technique (Pruessner et al., 1997). The CAR was computed by calculating the difference in cortisol levels between waking and 30 min after waking (Adam \& Kumari, 2009).

\section{Results}

Mean differences among study variables were examined for nativity status and annual household income, with no statistically significant variations observed. Cortisol readings were commensurate with those in past reports of Mexican American adolescents (Zeiders, Doane, \& Roosa, 2012) and migrant farmworkers (Clingerman \& Brown, 2012). As shown in Table 1, acculturative stress was significantly and negatively correlated with Anglo orientation. Preliminary analyses revealed that the bedtime cortisol levels for the sample were significantly and positively skewed, whereas those for Mexican orientation were negatively skewed. As recommended by Adam and Kumari (2009) and Tabachnick and Fidell (2013), square root transformations were conducted to approximate normal distributions and were entered into the main analyses. No significant outliers were observed. 
Table 1

Means, Standard Deviations, and Correlations of Main Study Variables

\begin{tabular}{|c|c|c|c|c|c|c|c|}
\hline Variables & 1 & 2 & 3 & 4 & 5 & 6 & 7 \\
\hline 1. Waking cortisol ( $\mu \mathrm{g} / \mathrm{dL})$ & - & .05 & .18 & $-.63^{* *}$ & -.08 & .26 & .08 \\
\hline 2. $30 \mathrm{~min}$ postwaking cortisol $(\mu \mathrm{g} / \mathrm{dL})$ & & - & -.11 & $.75^{* *}$ & -.37 & .12 & .14 \\
\hline 3. Bedtime cortisol $(\mu \mathrm{g} / \mathrm{dL})$ & & & - & -.24 & .24 & -.06 & -.29 \\
\hline 4. Cortisol awakening response (CAR) & & & & - & -.24 & -.08 & .06 \\
\hline 5. Acculturative stress & & & & & - & $-.50^{\circ}$ & .13 \\
\hline 6. Anglo orientation & & & & & & - & $-.44^{\dagger}$ \\
\hline 7. Mexican orientation & & & & & & & - \\
\hline $\bar{M}$ & .26 & .47 & .13 & .21 & 1.03 & 3.56 & 4.05 \\
\hline$S D$ & .11 & .13 & .14 & .17 & .62 & .98 & .86 \\
\hline Range & $.12-.46$ & $.18-.71$ & $.01-.44$ & $-.20-.42$ & $.08-2.60$ & $1.33-4.67$ & $1.83-5.00$ \\
\hline
\end{tabular}

${ }^{\dagger} p<.10 . \quad{ }^{*} p<.05 .{ }^{*} p<.01 .{ }^{\cdots} p<.001$.

Table 1. Means, Standard Deviations, and Correlations of Main Study Variables

\begin{tabular}{|l|l|l|l|l|l|l|l|}
\hline Variables & 1 & 2 & 3 & 4 & 5 & 6 \\
\hline 1. Waking cortisol $(\mu \mathrm{g} / \mathrm{dL})$ & - & .05 & .18 & $-.63^{* *}$ & -.08 & .26 & .08 \\
\hline 2. 30 min postwaking cortisol $(\mu \mathrm{g} / \mathrm{dL})$ & & - & -.11 & $.75^{* * *}$ & -.37 & .12 & .14 \\
\hline 3. Bedtime cortisol $(\mu \mathrm{g} / \mathrm{dL})$ & & & - & -.24 & .24 & -.06 & -.29 \\
\hline 4. Cortisol awakening response (CAR) & & & & - & -.24 & -.08 & .06 \\
\hline $\begin{array}{l}\text { Acculturative stress } \\
\text { Anglo orientation }\end{array}$ & & & & & - & $-.50^{*}$ & .13 \\
\hline 7. Mexican orientation & & & & & $-.44^{+}$ \\
\hline$M$ & & & & & & - \\
\hline SD & .26 & .47 & .13 & .21 & 1.03 & 3.56 & 4.05 \\
\hline Range & .11 & .13 & .14 & .17 & .62 & .98 & .86 \\
\hline
\end{tabular}

In order to examine the study hypotheses, a median split was used to divide acculturative stress into high and low groups. To test Hypothesis 1 , an independent samples $t$ test was conducted to examine mean differences based on high and low groups of acculturative stress. This analysis revealed a statistically significant variation such that the low acculturative stress group showed a higher CAR when compared to the high acculturative stress group, $M=.30(S D=.10)$ and $M=.13(S D=.18)$, respectively; $t(15)=2.34, p=.03$. As an estimate of effect size, Cohen's $d$ was 1.15, suggesting a large effect according to Cohen (1988), which is consistent with the results of previous work (Mangold et al., 2012). These results support the hypotheses of this study. Given that age, particularly as it pertains to menopausal symptoms, has been found to influence cortisol output (Fries et al., $\underline{2009}$ ), the sample was divided into two groups based on median split to examine potential variations in the CAR. No statistically significant differences were observed based on age.

To address Hypothesis 2, a repeated-measures analysis of variance was conducted to examine cortisol levels within high and low acculturative stress groups. Age and acculturation, in the form of the AOS and MOS, were entered as covariates. Mauchly's test of sphericity indicated that this assumption had not been violated, $\chi^{2}(2)=$ 2.11, $p=.35$. This analysis showed a main effect trending toward statistical significance in which cortisol levels may have changed across the three time points, $F(2,22)=3.01, p=.07, \eta^{2}=.22$. More importantly, a statistically significant interaction was observed that showed that changes in cortisol levels differed in the high and low acculturative stress groups, $F(2,22)=6.03, p=.008, \eta^{2}=.35$. Given the sample size, a post hoc power analysis was conducted and revealed excellent power $[(1-\beta)=0.99]$ given the calculated effect size, number of participants, and number of measurements (i.e., time points; $\alpha=.05$ ). As shown in Figure 1 , an illustration of the significant interaction shows comparable cortisol levels at waking, $t(15)=-.57, p=.58$; Cohen's $d=0.28$, but the 
high acculturative stress group had lower levels at 30 min postwaking, $t(15)=2.53, p=.02$; Cohen's $d=1.22$, and higher levels at bedtime, $t(8.31)=-2.11, p=.07$; Cohen's $d=1.06$. These findings seem to support the study hypotheses and suggest that participants who scored in the high acculturative stress group showed a flatter diurnal cortisol slope when compared to the low acculturative stress group.

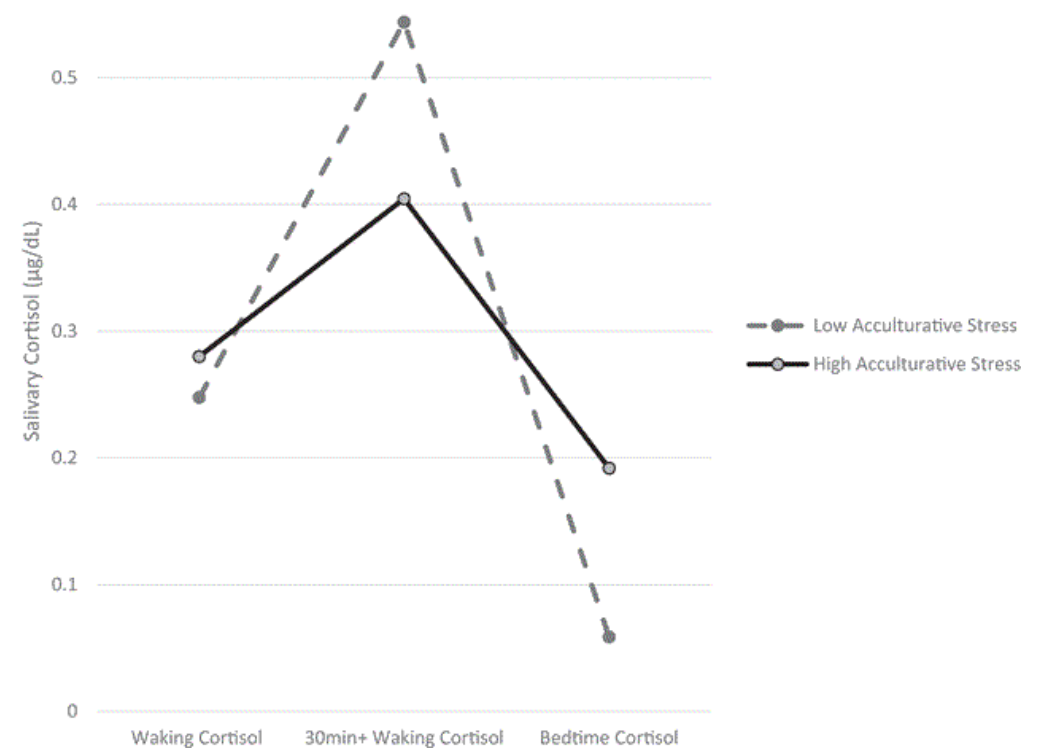

Figure 1. Diurnal cortisol response based on acculturative stress level. Nontransformed data shown.

\section{Discussion}

This study sought to better understand the biological indicators of stress as they relate to acculturative stress among Latina adults. Latina women are a group at high risk for experiencing increased stressors, both general and cultural, yet remain understudied in the broader empirical literature. Although some previous studies have examined ethnic discrimination and physiological responses (cf. Zeiders et al., 2012), to the best of our knowledge, this is the first study to examine acculturative stress and cortisol. Given the epidemiological research indicating that, for Latinos, increased exposure to the U.S. culture is associated with worse health outcomes (Alegría et al., 2007), acculturative stress could be a major risk factor that contributes to this phenomenon at a biological and psychological level. Overall, the findings of this initial exploratory study provide information regarding the potential role of cultural stressors in contributing to the biological stress response.

As expected, the findings suggest an attenuation effect in the CAR among the high acculturative stress group when compared to the low acculturative stress group. The CAR has been thought to indicate adrenocortical capacity, with decreased levels suggesting dysfunction and thus a vulnerability to physical and mental disorders (Meinlschmidt \& Heim, 2005), including posttraumatic stress disorder (Yehuda, 2002). Linking the experience of acculturative stress with other forms of chronic stress is consistent with a minority stress model (Myers, 2009).

The results of this study support the notion that individuals who endorse high levels of acculturative stress show a flatter diurnal cortisol response slope-namely, lower cortisol levels after waking yet higher cortisol levels in the evening-when compared to the low acculturative stress group. Such a pattern of diurnal cortisol response has been found in past research to represent a blunting of cortisol associated with chronic stress levels and the inability to build an effective HPA response (Clingerman \& Brown, 2012; Miller et al., 2007). It has been suggested that chronic and/or early exposure to stress can alter the HPA axis from a system of increased responsivity to one with a more diminished response (Heim, Ehlert, \& Hellhammer, 2000; Meinlschmidt \& Heim, 
2005). Furthermore, a flat diurnal cortisol pattern has been linked to instances of uncontrollable stress. However, it is difficult to determine whether the cortisol patterns observed in this study are due to an acute instance of acculturative stress or if they are a result of cumulative pressures.

Acculturative stress includes pressures to conform to mainstream values and ways of living as well as the pressure to retain the traditional culture. For Latina women, this balancing of cultural demands may be particularly burdensome given the gender role expectation that they be all-nurturing, pious, and all-sacrificing, termed marianismo, in order to place the needs of the family above their own (Santiago-Rivera, Arredondo, \& Gallardo-Cooper, 2002). It could be the case that numerous pressures stemming from different cultures have a marked influence on the individual's biological stress response.

There are several limitations to this study that should be noted. First, as stated previously, this is an initial exploratory study; given the small sample size, conclusions must be tempered and interpreted with caution. Still, this study has addressed a gap in the empirical research by investigating acculturative stress while accounting for acculturation and age in relation to commonly used biological markers of stress. Further research should examine these processes with a larger sample size to allow for sophisticated analyses such as multivariate repeated-measures modeling. Second, the sample characteristics, beyond sample size, restrict the generalization of the results to the broader Latino community - that is, the participants consisted of women who were well educated and from middle- to high-income socioeconomic backgrounds, which may influence the nature and type of stressors experienced. Still, this sample of Latina women represents a growing subset of the population, and these results begin to inform the complex and multilayered lived experiences of these individuals. In terms of cortisol sampling, three time points may have limited the characterization of the diurnal slope; thus, future work should seek to sample four to five time points a day across multiple days. Furthermore, assessing the CAR with two time points does not allow for the calculation of area under curve with respect to ground, which determines the peak of awakening cortisol levels. Also, this study did not assess menstrual cycle or body mass index, both of which have been found to influence cortisol output. Finally, unlike the assessment of acculturation, the measurement of acculturative stress did not include separate scores to account for bidimensionality.

Despite these limitations, this initial exploratory study provides information regarding the potential interplay of culture and biology among Latinos and begins to address the role of acculturative stress. Future research should continue to address acculturative stress and the related physiological consequences to replicate the current findings and to expand this line of inquiry to the larger community. Along these lines, assessing the complexity of HPA axis functioning should include the measurement of ACTH production and CRF secretion and indicators of allostatic load. Analyses that examine the various types of acculturative stress are needed to refine the nature of these experiences and their contribution to biological responses.

\section{References}

Adam, E. K., Heissel, J. A., Zeiders, K. H., Richeson, J. A., Ross, E. C., Ehrlich, K. B., . . Eccles, J. S. (2015). Developmental histories of perceived racial discrimination and diurnal cortisol profiles in adulthood: A 20-year prospective study. Psychoneuroendocrinology, 62, 279-291. 10.1016/j.psyneuen.2015.08.018

Adam, E. K., \& Kumari, M. (2009). Assessing salivary cortisol in large-scale, epidemiological research. Psychoneuroendocrinology, 34, 1423-1436. 10.1016/j.psyneuen.2009.06.011

Alegría, M., Mulvaney-Day, N., Torres, M., Polo, A., Cao, Z., \& Canino, G. (2007). Prevalence of psychiatric disorders across Latino subgroups in the United States. American Journal of Public Health, 97, 68-75. 10.2105/AJPH.2006.087205

American Psychological Association. (2016). Stress in America: The impact of discrimination. Washington, DC: Author. 
Bauman, S. (2005). The reliability and validity of the Brief Acculturation Rating Scale for Mexican Americans-II for children and adolescents. Hispanic Journal of Behavioral Sciences, 27, 426-441.

10.1177/0739986305281423

Berry, J. W. (2006). Acculturative stress. In P. T. P.Wong \& L. C. S.Wong (Eds.), Handbook of multicultural perspectives on stress and coping (pp. 287-298). Langley, British Columbia, Canada: Springer. 10.1007/0387-26238-5_12

Center for Economic Development. (2016). Latino Milwaukee: A statistical portrait. Milwaukee, WI: Greater Milwaukee Foundation.

Clingerman, E. M., \& Brown, A. (2012). Stress in migrant farmworkers during premigration. Biological Research for Nursing, 14, 27-37. 10.1177/1099800410396703

Clow, A., Hucklebridge, F., Stalder, T., Evans, P., \& Thorn, L. (2010). The cortisol awakening response: More than a measure of HPA axis function. Neuroscience and Biobehavioral Reviews, 35, 97-103. 10.1016/j.neubiorev.2009.12.011

Cohen, J. (1988). Statistical power analysis for the behavioral sciences (2nd ed.). Hillsdale, NJ: Erlbaum.

Cuéllar, I., Arnold, B., \& Maldonado, R. (1995). Acculturation Rating Scale for Mexican Americans-II: A revision of the original ARSMA scale. Hispanic Journal of Behavioral Sciences, 17, 275-304. 10.1177/07399863950173001

D’Anna-Hernandez, K. L., Hoffman, M. C., Zerbe, G. O., Coussons-Read, M., Ross, R. G., \& Laudenslager, M. L. (2012). Acculturation, maternal cortisol, and birth outcomes in women of Mexican descent. Psychosomatic Medicine, 74, 296-304. 10.1097/PSY.0b013e318244fbde

de Castro, A. B., Voss, J. G., Ruppin, A., Dominguez, C. F., \& Seixas, N. S. (2010). Stressors among Latino day laborers: A pilot study examining allostatic load. AAOHN Journal, 58, 185-196.

Dowd, J. B., Simanek, A. M., \& Aiello, A. E. (2009). Socio-economic status, cortisol and allostatic load: A review of the literature. International Journal of Epidemiology, 38, 1297-1309. 10.1093/ije/dyp277

Fries, E., Dettenborn, L., \& Kirschbaum, C. (2009). The cortisol awakening response (CAR): Facts and future directions. International Journal of Psychophysiology, 72, 67-73. 10.1016/j.ijpsycho.2008.03.014

Galanti, G.-A. (2003). The Hispanic family and male-female relationships: An overview. Journal of Transcultural Nursing, 14, 180-185. 10.1177/1043659603014003004

Gallagher-Thompson, D., Shurgot, G. R., Rider, K., Gray, H. L., McKibbin, C. L., Kraemer, H. C., . . Thompson, L. W. (2006). Ethnicity, stress, and cortisol function in Hispanic and non-Hispanic white women: A preliminary study of family dementia caregivers and noncaregivers. The American Journal of Geriatric Psychiatry, 14, 334-342. 10.1097/01.JGP.0000206485.73618.87

Gonzales, N. A., Germán, M., \& Fabrett, F. C. (2012). U.S. Latino youth. In E. C.Chang \& C. A.Downey (Eds.), Handbook of race and development of mental health (pp. 259-278). New York, NY: Springer. 10.1007/978-1-4614-0424-8_15

Gozansky, W. S., Lynn, J. S., Laudenslager, M. L., \& Kohrt, W. M. (2005). Salivary cortisol determined by enzyme immunoassay is preferable to serum total cortisol for assessment of dynamic hypothalamic-pituitaryadrenal axis activity. Clinical Endocrinology, 63, 336-341. 10.1111/j.1365-2265.2005.02349.x

Heim, C., Ehlert, U., \& Hellhammer, D. H. (2000). The potential role of hypocortisolism in the pathophysiology of stress-related bodily disorders. Psychoneuroendocrinology, 25, 1-35. 10.1016/S0306-4530(99)00035-9

Hovey, J. D., \& Magana, C. G. (2002). Psychosocial predictors of anxiety among immigrant Mexican migrant farmworkers: Implications for prevention and treatment. Cultural Diversity and Ethnic Minority Psychology, 8, 274-289.

Jackson, J. S., Knight, K. M., \& Rafferty, J. A. (2010). Race and unhealthy behaviors: Chronic stress, the HPA axis, and physical and mental health disparities over the life course. American Journal of Public Health, 100, 933-939.

Juster, R.-P., McEwen, B. S., \& Lupien, S. J. (2010). Allostatic load biomarkers of chronic stress and impact on health and cognition. Neuroscience and Biobehavioral Reviews, 35, 2-16.

10.1016/j.neubiorev.2009.10.002 
Kudielka, B. M., Hellhammer, D. H., \& Wüst, S. (2009). Why do we respond so differently? Reviewing determinants of human salivary cortisol responses to challenge. Psychoneuroendocrinology, 34, 2-18. 10.1016/j.psyneuen.2008.10.004

Kudielka, B. M., \& Kirschbaum, C. (2005). Sex differences in HPA axis responses to stress: A review. Biological Psychology, 69, 113-132. 10.1016/j.biopsycho.2004.11.009

Mangold, D., Marino, E., \& Javors, M. (2011). The cortisol awakening response predicts subclinical depressive symptomatology in Mexican American adults. Journal of Psychiatric Research, 45, 902-909. 10.1016/j.jpsychires.2011.01.001

Mangold, D., Mintz, J., Javors, M., \& Marino, E. (2012). Neuroticism, acculturation and the cortisol awakening response in Mexican American adults. Hormones and Behavior, 61, 23-30. 10.1016/j.yhbeh.2011.09.009

Mangold, D., Wand, G., Javors, M., \& Mintz, J. (2010). Acculturation, childhood trauma and the cortisol awakening response in Mexican-American adults. Hormones and Behavior, 58, 637-646. 10.1016/j.yhbeh.2010.06.010

McEwen, B. S. (1998). Stress, adaptation, and disease: Allostasis and allostatic load. Annals of the New York Academy of Sciences, 840, 33-44. 10.1111/j.1749-6632.1998.tb09546.x

Meinlschmidt, G., \& Heim, C. (2005). Decreased cortisol awakening response after early loss experience. Psychoneuroendocrinology, 30, 568-576. 10.1016/j.psyneuen.2005.01.006

Miller, G. E., Chen, E., \& Zhou, E. S. (2007). If it goes up, must it come down? Chronic stress and the hypothalamic-pituitary-adrenocortical axis in humans. Psychological Bulletin, 133, 25-45. 10.1037/00332909.133.1.25

Myers, H. F. (2009). Ethnicity- and socio-economic status-related stresses in context: An integrative review and conceptual model. Journal of Behavioral Medicine, 32, 9-19. 10.1007/s10865-008-9181-4

Pascoe, E. A., \& Smart Richman, L. (2009). Perceived discrimination and health: A meta-analytic review. Psychological Bulletin, 135, 531-554. 10.1037/a0016059

Pruessner, J. C., Wolf, O. T., Hellhammer, D. H., Buske-Kirschbaum, A., von Auer, K., Jobst, S., . . Kirschbaum, C. (1997). Free cortisol levels after awakening: A reliable biological marker for the assessment of adrenocortical activity. Life Sciences, 61, 2539-2549. 10.1016/S0024-3205(97)01008-4

Rodriguez, N., Myers, H. F., Mira, C. B., Flores, T., \& Garcia-Hernandez, L. (2002). Development of the Multidimensional Acculturative Stress Inventory for adults of Mexican origin. Psychological Assessment, 14, 451-461. 10.1037/1040-3590.14.4.451

Ruiz, R. J., Pickler, R. H., Marti, C. N., \& Jallo, N. (2013). Family cohesion, acculturation, maternal cortisol, and preterm birth in Mexican-American women. International Journal of Women's Health, 5, 243-252. 10.2147/IJWH.S42268

Ruiz, R. J., Stowe, R. P., Brown, A., \& Wommack, J. (2012). Acculturation and biobehavioral profiles in pregnant women of Hispanic origin: Generational differences. Advances in Nursing Science, 35(3), E1-E10. 10.1097/ANS.0b013e3182626199

Santiago-Rivera, A. L., Arredondo, P., \& Gallardo-Cooper, M. (2002). Counseling Latinos and la familia: A practical guide. Thousand Oaks, CA: Sage.

Skinner, M. L., Shirtcliff, E. A., Haggerty, K. P., Coe, C. L., \& Catalano, R. F. (2011). Allostasis model facilitates understanding race differences in the diurnal cortisol rhythm. Development and Psychopathology, 23, 1167-1186. 10.1017/S095457941100054X

Squires, E. C., McClure, H. H., Martinez, C. R., Jr., Eddy, J. M., Jiménez, R. A., Isiordia, L. E., \& Snodgrass, J. J. (2012). Diurnal cortisol rhythms among Latino immigrants in Oregon, USA. Journal of Physiological Anthropology, 31, 19-29. 10.1186/1880-6805-31-19

Suglia, S. F., Staudenmayer, J., Cohen, S., Enlow, M. B., Rich-Edwards, J. W., \& Wright, R. J. (2010). Cumulative stress and cortisol disruption among Black and Hispanic pregnant women in an urban cohort. Psychological Trauma: Theory, Research, Practice, and Policy, 2, 326-334. 10.1037/a0018953

Tabachnick, B. G., \& Fidell, L. S. (2013). Using multivariate statistics (6th ed.). Upper Saddle River, NJ: Pearson.

Torres, L. (2010). Predicting levels of Latino depression: Acculturation, acculturative stress, and coping. Cultural Diversity and Ethnic Minority Psychology, 16, 256-263. 10.1037/a0017357 
Torres, L., Driscoll, M. W., \& Voell, M. (2012). Discrimination, acculturation, acculturative stress, and Latino psychological distress: A moderated mediational model. Cultural Diversity and Ethnic Minority Psychology, 18, 17-25. 10.1037/a0026710

U.S. Census Bureau. (2013). State and county quick facts: United States. Retrieved from https://www.census.gov/quickfacts/

Yehuda, R. (2002). Current status of cortisol findings in post-traumatic stress disorder. Psychiatric Clinics of North America, 25, 341-368. 10.1016/S0193-953X(02)00002-3

Zeiders, K. H., Doane, L. D., \& Roosa, M. W. (2012). Perceived discrimination and diurnal cortisol: Examining relations among Mexican American adolescents. Hormones and Behavior, 61, 541-548. 10.1016/j.yhbeh.2012.01.018

Submitted: August 3, 2016 Revised: January 16, 2017 Accepted: January 25, 2017 